\title{
Erratum: Efficient orbital angular momentum transfer between plasmons and free electrons [Phys. Rev. B 98, 045424 (2018)]
}

Wei Cai, Ori Reinhardt, Ido Kaminer, and F. Javier García de Abajo

Q (Received 2 February 2019; published 14 February 2019)

DOI: 10.1103/PhysRevB.99.079904

The explicit factor of $\gamma$ should be removed from Eqs. (4b), (A1), and (A2) as well as from the equation for $g$ given three lines below Eq. (6), so they become

$$
\begin{aligned}
\beta & =\frac{e}{\hbar \omega_{\mathrm{p}}} \int_{-\infty}^{\infty} d z \mathcal{E}_{z}(\mathbf{R}, z) e^{-i \omega_{\mathrm{p}} z / v}, \\
(\mathbf{v} \cdot \mathbf{\nabla}+\partial / \partial t) \phi & =\frac{-i e \mathbf{v}}{\hbar c} \cdot \overrightarrow{\mathcal{A}} \phi, \\
\phi(\mathbf{r}, t) & =\phi_{0}(\mathbf{r}-\mathbf{v} t) \exp \left[\frac{-i e \mathbf{v}}{\hbar c} \cdot \int_{-\infty}^{t} d t^{\prime} \overrightarrow{\mathcal{A}}\left(\mathbf{r}+\mathbf{v} t^{\prime}-\mathbf{v} t, t^{\prime}\right)\right], \\
g & =e \mathcal{E}_{0} \lambda_{\mathrm{p}} / 2 \pi \hbar \omega_{\mathrm{p}},
\end{aligned}
$$

respectively. These changes do not affect the results and conclusions of the paper, except that the values of $\eta$ represented in Fig. 2(b) have to be divided by $\gamma \approx 1.002,1.02$, and 1.2 for $E_{0}=1,10$, and $100 \mathrm{keV}$, respectively. 